\title{
Special issue: selected papers from the 21st international symposium on temporal representations and reasoning (TIME-2014)
}

\author{
Davide Bresolin $^{1}$ • Guido Sciavicco ${ }^{2}$
}

Received: 2 February 2016 / Accepted: 3 February 2016 / Published online: 4 March 2016

(C) Springer-Verlag Berlin Heidelberg 2016

Time representation and reasoning is a challenging research field of Computer Science, with a recognized impact in artificial intelligence, databases and formal verification. Among the several formalisms that have been studied for temporal reasoning, two of them are particularly interesting, namely, temporal logics based on intervals, and temporal networks. Intervalbased languages are involved at many levels in different applications, such as planning and management of medical information, verification of hardware circuits, model-checking and requirement analysis of hybrid systems. Non-trivial problems rise at every level: expressive power of the languages, decidability and un-decidability of logics at the first-order and at the modal levels, computational complexity of automatic and semi-automatic deduction techniques, and axiomatic systems. Similarly, various kinds of temporal networks have been proposed in the literature including: temporal constraint networks, simple temporal networks (also with uncertainty, preferences, and probabilities), disjunctive temporal networks, and conditional temporal networks. For each kind of network, different notions of consistency and/or controllability have been defined, and algorithms for determining whether networks have the desired property have been presented. Some algorithms have been proven to be polynomial, others are exponential. Algorithms for managing the execution of various kinds of temporal networks have also been proposed.

This special issue contains six papers selected from the proceedings of the 21 st international symposium on temporal representations and reasoning (TIME-2014), and, in particular, from the special tracks on interval temporal logics and temporal networks. The 21 st edition of the TIME symposium has been chaired by Amedeo Cesta (CNR, Italy), Carlo Combi (University of Verona, Italy), and Francoise Laroussinie (University of Paris 7, France), and it took place in Verona, from the 8th to the 10th of September, 2014.

$\bowtie$ Davide Bresolin

davide.bresolin@unibo.it

Guido Sciavicco

guido@um.es

1 Department of Computer Science and Engineering, University of Bologna, Bologna, Italy

2 Department of Information Engineering and Communications, University of Murcia, Murcia, Spain 
Carlo Combi and Pietro Sala, in Mining Approximate Interval-based Temporal Dependencies, analyze the complexity of finding approximate interval-based temporal functional dependencies in the context of temporal databases; they also show how the complexity of the problem significantly changes depending on the considered interval relations.

Alberto Molinari, Angelo Montanari, Aniello Murano, Giuseppe Perelli and Adriano Peron, in Checking Interval Properties of Computations, give a formalization of the model checking problem in an interval setting: first they provide and interpretation of formulas of the Halpern and Shoham's interval logic over finite Kripke structures, and then they prove that the model checking problem is decidable via a suitable small model theorem.

Angelo Montanari, Marco Pazzaglia and Pietro Sala, in Metric Propositional Neighborhood Logic with an Equivalence Relation, study the effect of adding an equivalence relation to the so-called propositional neighborhood logic, proving that such an extension does not change the complexity of the finite satisfiability problem in the non-metric case, while it increases the complexity from NEXPTIME to EXPSPACE in the metric case.

Marta Cialdea Mayer, Andrea Orlandini and Alessandro Umbrico, in Planning and Execution with Flexible Timelines: a Formal Account, present a formal account of flexible timelines with the aim of providing a general semantics for planning concepts such as domains, goals, problems, constraints, and flexible plans; sources of uncertainty are modeled and taken into account in the characterization of valid plans and in the formal definition of plan controllability.

Andrea Micheli, Alessandro Cimatti, Luke Hunsberger, Roberto Posenato and Marco Roveri, in Dynamic Controllability via Timed Game Automata, introduce a new approach to determine the dynamic controllability of a very expressive class of temporal networks that accommodates observation nodes and disjunctive constraints; the approach is based on encoding the dynamic controllability problem into a reachability game for Timed Game Automata and it has immediate applications in the design and analysis of workflow models being developed to automate business processes, including workflows in the health-care domain.

Finally, Mikael Nilsson, Jonas Kvarnström, and Patrick Doherty, in Efficient Processing of Simple Temporal Networks with Uncertainty-Algorithms for Dynamic Controllability Verification, consider a recent incremental algorithm that can verify dynamic controllability of simple temporal network with uncertainty, and improve its efficiency so that nodes are not reprocessed; this improvement leads to a lower worst case complexity. 